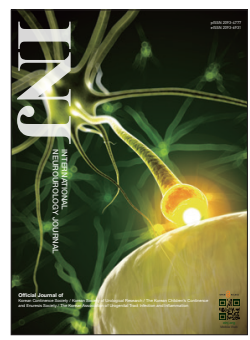

\title{
Neural Repair and Tissue Regeneration
}

\author{
Chang-Ju Kim (id https://orcid.org/0000-0003-4749-5795 \\ Department of Physiology, College of Medicine, Kyung Hee University, Seoul, Korea \\ E-mail: changju@khu.ac.kr
}

This special issue of the International Neurourology Journal recruited papers on the theme "neural repair and tissue regeneration." The articles published in this issue provide an opportunity to reflect on the mechanisms underlying nerve damage and treatment, as well as treatments or candidate substances for tissue regeneration.

Kim et al. [1] wrote a review paper on the therapeutic potential of stem cell therapy for neurogenic bladder resulting from spinal cord injury. It is nonetheless true that many questions remain about the stability and effectiveness of stem cell therapy after preclinical and clinical studies. Further research is needed to prove the therapeutic effectiveness of stem cell therapy. In the paper of Jang et al. [2], after a crush injury was applied to the sciatic nerve of rats, dexmedetomidine was administered to the injury site to analyze the therapeutic effect of dexmedetomidine. Dexmedetomidine enhanced the locomotor function of rats with sciatic crushed nerve injury and decreased the expression of c-Fos, which was increased by pain. This mechanism of dexmedetomidine suggests that it may have a therapeutic effect on intractable urogenital ulcerations. Hwang and Namgung [3] studied the effect of cyclin-dependent kinase 5 (Cdk5) on axonal regeneration after sciatic nerve crushed injury, and found that Cdk5 phosphorylated STAT3 to promote axonal regeneration after the injury. Therefore, STAT3 is thought to be an intrinsic factor that promotes axonal regeneration in peripheral nerve injuries. Two papers investigated the effects of exercise on incurable diseases of the nervous system. Hong et al. [4] evaluated the effect of treadmill exercise after inducing photothrombotic stroke in mice. After stroke, treadmill exercise increased synaptic plasticity and nerve cell production, preventing motor and memory loss. Kim et al. [5] investigated the neuroprotective action of swimming exercise on autoimmune encephalomyelitis rats, an animal model of multiple sclerosis. Swimming exercise reduced motor dysfunction by inhibiting demyelination and the expression of pro-inflammatory cytokines and inflammatory mediators. Ban et al. [6] established that glycyrrhizic acid, the main component of Glycyrrhiza uralensis, exerted neuroprotective actions against scopolamine-induced cognitive impairment, as the administration of glycyrrhizic acid improved scopolamine-induced cognitive impairment. Hwang et al. [7] demonstrated that the combination of polydeoxyribonucleotide and pirfenidone was effective in treating acute respiratory distress syndrome, as polydeoxyribonucleotide and pirfenidone combination therapy exerted an anti-inflammatory effect on acute respiratory distress syndrome and also inhibited fibrosis.

Nerve repair and tissue regeneration are the most important topics in the urology field. Promoting repair and regeneration for ulcers in the bladder and urethra or partial deficits in the kidneys may be the ultimate goal of treatment. By recruiting papers for this special issue, we tried to overcome the limitations of treatments of urological diseases by soliciting insights into treatment approaches for other diseases.

Even under the circumstances of the coronavirus crisis, we are responding to the challenge to identify new therapeutic techniques or mechanisms for intractable urological diseases. We appreciate the authors for contributing these valuable articles. 
- Conflict of Interest: No potential conflict of interest relevant to this article was reported.

\section{REFERENCES}

1. Kim SJ, Cho YS, Kim KH. Stem cell therapy for neurogenic bladder after spinal cord injury: clinically possible? Int J Neurourol J 2020; 24(Suppl 1):S3-10.

2. Jang MS, Han JH, Kim DO, Woo G, Kwon JK, Chung JY, et al. Dexmedetomidine improves locomotor function and alleviates thermal hyperalgesia following sciatic nerve crush injury in rats. Int J Neurourol J 2020;24(Suppl 1):S11-8 .

3. Hwang J, Namgung U. Cdk5 phosphorylation of STAT3 in dorsal root ganglion neurons is involved in promoting axonal regeneration after peripheral nerve injury. Int J Neurourol J 2020;24(Suppl 1):S19-27.

4. Hong M, Kim M, Kim TW, Park SS, Kim MK, Park YH, et al.
Treadmill exercise improves motor function and short-term memory by enhancing synaptic plasticity and neurogenesis in photothrombotic stroke mice. Int J Neurourol J 2020;24(Suppl 1):S28-38.

5. Kim JY, Yi ES, Lee H, Kim JS, Jee YS, Kim SE, et al. Swimming exercise ameliorates symptoms of myelin oligodendrocyte glycoprotein-induced experimental autoimmune encephalomyelitis by inhibiting inflammation and demyelination in rats. Int J Neurourol J 2020;24(Suppl 1):S39-47.

6. Ban JY, Park HK, Kim SK. Effect of glycyrrhizic acid on scopolamine-induced cognitive impairment in mice. Int J Neurourol J 2020;24(Suppl 1):S48-55.

7. Hwang JJ, Ko IG, Jin JJ, Hwang L, Kim SH, Jeon JW, et al. Combination therapy with polydeoxyribonucleotide and pirfenidone alleviates symptoms of acute respiratory distress syndrome in human lung epithelial A549 cells. Int J Neurourol J 2020;24(Suppl 1):S5664. 